\title{
Stenting versus coronary artery bypass grafting for unprotected left main coronary artery disease: A meta-analysis of comparative studies
}

\author{
Hisato Takagi, MD, PhD, Norikazu Kawai, MD, and Takuya Umemoto, MD, PhD, Shizuoka, Japan
}

Although coronary artery bypass grafting (CABG) is currently recommended to treat unprotected left main coronary artery (LMCA) disease, the treatment of this disorder by percutaneous coronary intervention represents a considerable challenge for interventional cardiologists. ${ }^{1}$ Several studies have analyzed the treatment of unprotected LMCA disease with $\mathrm{CABG}$ versus percutaneous coronary intervention with stents (PCI-S), but no meta-analyses of these studies have been conducted to date. To compare the treatment of unprotected LMCA disease with PCI-S versus CABG, we performed a meta-analysis of comparative studies for prevention of death, repeated revascularization, and major adverse cardiac and cerebrovascular events (MACCEs) at follow-up.

\section{MATERIALS AND METHODS}

All comparative studies of PCI-S (with bare metal or drug-eluting stents) versus CABG (conventional or off-pump CABG) enrolling patients with unprotected LMCA disease were identified by means of a 2-level search strategy. First, a public domain database (MEDLINE) was searched with a web-based search engine (PubMed). Second, relevant studies were identified through a manual search of secondary sources, including references of initially identified articles and a search of reviews and commentaries. The MEDLINE database was searched from January 1966 to March 2008. MeSH keywords included coronary artery bypass; angioplasty, transluminal, percutaneous coronary; and stents. Studies considered for inclusion met the following criteria: the study design was comparative (randomized controlled or nonrandomized observational); the study population consisted of patients with unprotected LMCA disease; patients were assigned to undergo PCI-S versus CABG; and main outcomes included death, repeated revascularization, or MACCEs (death, myocardial infarction, stroke, or repeated revascularization) at follow-up. Data regarding detailed inclusion criteria, stent type, duration of follow-up, and negative outcomes (death, repeated revascularization, and MACCEs) at follow-up were abstracted as available from each individual study. Adjusted risk estimates for nonrandomized controlled comparisons and crude risk ratios for randomized controlled comparisons were pooled after logarithmic transformation according to a random-effects model with generic inverse variance weighting. Interstudy heterogeneity was analyzed by means of standard $\chi^{2}$ tests.

From the Department of Cardiovascular Surgery, Shizuoka Medical Center, Shizuoka, Japan.

Received for publication March 7, 2008; accepted for publication June 15, 2008.

Address for reprints: Hisato Takagi, MD, PhD, Department of Cardiovascular Surgery,

Shizuoka Medical Center, 762-1 Nagasawa, Shimizu-cho, Sunto-gun, Shizuoka

411-8611, Japan (E-mail: kfgth973@ybb.ne.jp).

J Thorac Cardiovasc Surg 2009;137:e54-7

$0022-5223 / \$ 36.00$

Copyright (c) 2009 by The American Association for Thoracic Surgery

doi:10.1016/j.jtcvs.2008.06.006

\section{RESULTS}

Our search identified 6 comparative studies ${ }^{1-6}$ of PCI-S versus $\mathrm{CABG}$ that had enrolled patients with unprotected LMCA disease. These included 1 randomized controlled trial $^{2}$ and 5 nonrandomized observational studies. ${ }^{1,3-6} \mathrm{We}$ excluded 2 nonrandomized observational studies, by Brener and colleagues (2008) and Sanmartín and associates (2007), because adjusted risk estimates could not be abstracted. In total, our meta-analysis included data on 2181 patients with unprotected LMCA disease assigned to undergo PCI-S $(n=1006)$ or CABG $(n=1175)$. The baseline patient and procedural characteristics are summarized in Table 1. For death at follow-up, 3 studies $^{1,2,4}$ demonstrated a statistically nonsignificant benefit of PCI-S relative to CABG, whereas 2 studies $^{3,6}$ demonstrated a statistically nonsignificant benefit of CABG relative to PCI-S. Pooled analysis of the 5 studies reporting this outcome demonstrated no significant difference in death rate between PCI-S and CABG $(P=.97$; Figure $1, A)$. There was no significant interstudy heterogeneity of results $(P=.36)$. For repeated revascularization at follow-up, all 4 studies reporting this outcome $^{1-3,6}$ demonstrated a statistically significant benefit of CABG relative to PCI-S. Pooled analysis of these 4 studies demonstrated a statistically significant increase in repeated revascularization with PCI-S relative to CABG $(P<$ .00001 ; Figure $1, B)$. There was no significant interstudy heterogeneity of results $(P=.25)$. For MACCEs at followup, 2 studies ${ }^{1,5}$ demonstrated a statistically nonsignificant benefit of PCI-S relative to CABG, whereas 1 study $^{2}$ demonstrated a statistically nonsignificant benefit of $\mathrm{CABG}$ relative to PCI-S. Pooled analysis of the 3 studies that reported MAC$\mathrm{CEs}$ as an outcome demonstrated a statistically nonsignificant reduction in MACCEs with PCI-S relative to CABG $(P=.32$; Figure $1, C)$. There was no significant interstudy heterogeneity of results $(P=.07)$. When data were pooled with a fixed-effects model, the overall results of our analysis were not substantively altered.

\section{DISCUSSION}

On the basis of this meta-analysis, CABG is likely to provide better results than PCI-S not in terms of death and MACCEs but in terms of repeated revascularization at 6 months to 3 years of follow-up. Main limitations of our analysis are the following: only a single randomized, controlled trial was included, and it was small; both bare metal and drug-eluting stents were used in the PCI-S group; both conventional and off-pump CABG were performed in the 
TABLE 1. Baseline patient and procedural characteristics

\begin{tabular}{|c|c|c|c|c|c|c|}
\hline & $\begin{array}{c}\text { Seung } \\
\text { et } \mathrm{al}, \mathbf{2 0 0 8}^{3}\end{array}$ & $\begin{array}{c}\text { Buszman } \\
\text { et al, 2008 }\end{array}$ & $\begin{array}{l}\text { Palmerini } \\
\text { et al, 2007 }\end{array}$ & $\begin{array}{c}\text { Chieffo } \\
\text { et al, 2006 }\end{array}$ & $\begin{array}{c}\text { Lee } \\
\text { et al, } 2006^{5}\end{array}$ & $\begin{array}{l}\text { Palmerini } \\
\text { et al, 2006 }\end{array}$ \\
\hline Design & Observational & Randomized & Observational & Observational & Observational & Observational \\
\hline Follow-up & $\begin{array}{r}1017 \mathrm{~d}^{*}(\mathrm{IQR} 688- \\
1451 \mathrm{~d}), 1152 \mathrm{~d}^{*} \\
(\mathrm{IQR} 681-1590 \mathrm{~d})\end{array}$ & $1 \mathrm{y}$ & $2 \mathrm{y}$ & $1 \mathrm{y}$ & $\begin{array}{l}5.6 \pm 3.9 \mathrm{mo} \\
6.7 \pm 6.2 \mathrm{mo}\end{array}$ & $\begin{array}{c}417 \mathrm{~d}^{*}(2-830 \mathrm{~d}) \\
430 \mathrm{~d} *(105- \\
730 \mathrm{~d})\end{array}$ \\
\hline$P$ value $\dagger$ & & & & & .26 & .439 \\
\hline Patients (No.) & $542, \ddagger 542 \ddagger$ & 52,53 & 98,161 & 107,142 & 50,123 & 157,154 \\
\hline Age (y) & $\begin{array}{l}\text { 64* (IQR 56-71), } \\
\text { 64*(IQR 56-70) }\end{array}$ & $\begin{array}{r}60.6 \pm 10.5 \\
61.3 \pm 8.4\end{array}$ & $\begin{array}{l}81 *(75-88), \\
78 *(75-88)\end{array}$ & $\begin{array}{r}63.6 \pm 10.3 \\
67.5 \pm 9.7\end{array}$ & $\begin{array}{l}72 \pm 15 \\
70 \pm 10\end{array}$ & $\begin{array}{r}73.0 \pm 10.9 \\
69.3 \pm 9.5\end{array}$ \\
\hline$P$ value $\dagger$ & .41 & .69 & $<.001$ & .002 & .33 & .002 \\
\hline Male $(\%)$ & $71.6 \%, 71.2 \%$ & $60 \%, 73 \%$ & $54 \%, 66 \%$ & NR & $50 \%, 76 \%$ & $70.1 \%, 76.0 \%$ \\
\hline$P$ value $\dagger$ & .95 & .13 & .06 & & $<.01$ & .296 \\
\hline $\begin{array}{l}\text { Ejection } \\
\quad \text { fraction }(\%)\end{array}$ & $\begin{array}{c}61 \% *(\text { IQR } 54 \%- \\
66 \%), 61 \% * \\
(\text { IQR } 55 \%-66 \%)\end{array}$ & $\begin{array}{r}53.5 \% \pm 10.7 \% \\
53.7 \% \pm 6.7 \%\end{array}$ & $\begin{array}{c}50 \% *(20 \%-70 \%) \\
53 \% *(25 \%- \\
78 \%)\end{array}$ & $\begin{array}{r}52.0 \% \pm 10.4 \% \\
52.2 \% \pm 11.4 \%\end{array}$ & $\begin{array}{r}51 \% \pm 15 \% \\
52 \pm 10 \%\end{array}$ & $\begin{array}{r}51.8 \pm 13.8 \% \\
54.6 \pm 12 \%\end{array}$ \\
\hline$P$ value $\dagger$ & .62 & .86 & .68 & .91 & .64 & .069 \\
\hline \multicolumn{7}{|l|}{$\begin{array}{l}\text { Clinical } \\
\text { presentation }\end{array}$} \\
\hline $\begin{array}{l}\text { Stable angina } \\
(\%)\end{array}$ & $29.2 \%, 28.4 \% \S$ & $\begin{array}{c}\text { CCS class } 3.1 \pm 1.0 \\
2.8 \pm 1.0\end{array}$ & NR & NR & $34 \%, 55 \%$ & $26.8 \%, 27.9 \%$ \\
\hline$P$ value $\dagger$ & & .17 & & & NR & .917 \\
\hline $\begin{array}{l}\text { Unstable } \\
\text { angina (\%) }\end{array}$ & $57.4 \%, 57.9 \% \S$ & & $\begin{array}{l}\text { Acute coronary } \\
\text { syndrome } 77 \% \text {, } \\
52 \%\end{array}$ & $31.8 \%, 21.8 \%$ & $46 \%, 25 \%$ & $\begin{array}{l}\text { Braunwald class IB }+ \\
\quad \text { IIB } 13.4 \%, 20.1 \% \\
\quad(P=.149) ; \text { IIIB } \\
\quad 13.4 \%, 7.8 \%(P \\
\quad=.157)\end{array}$ \\
\hline$P$ value $\dagger$ & & & $<.001$ & .08 & NR & \\
\hline $\begin{array}{l}\text { Myocardial } \\
\quad \text { infarction (\%) }\end{array}$ & $10.7 \%,\|11.1 \%\| \S$ & & & NR & $20 \%, 20 \%$ & $39.5 \%$, $31.2 \%$ ๆ \\
\hline$P$ value $\dagger$ & & & & & NR & .157 \\
\hline \multicolumn{7}{|l|}{ Surgical risk } \\
\hline EuroSCORE & NR & $\begin{array}{l}3.3 \pm 2.3 \\
3.5 \pm 2.3\end{array}$ & $\begin{array}{l}8 *(4-18) \\
7 *(3-14)\end{array}$ & $\begin{array}{r}4.4 \pm 3.6 \\
4.3 \pm 3.4\end{array}$ & NR & $\begin{array}{l}6^{*}(0-18) \\
5^{*}(0-14)\end{array}$ \\
\hline$P$ value $\dagger$ & & .65 & $<.001$ & .85 & & .032 \\
\hline Parsonnet score & & NR & $\begin{array}{c}22.5 *(12.5-38.5) \\
14 *(5-36)\end{array}$ & NR & $\begin{array}{r}18.3 \pm 10.9 \\
13.7 \pm 9.7\end{array}$ & $\begin{array}{c}16.5 *(2.5-37), 12.5^{*} \\
(2-36)\end{array}$ \\
\hline$P$ value $\dagger$ & & & $<.001$ & & $<.01$ & .004 \\
\hline \multicolumn{7}{|l|}{$\begin{array}{l}\text { Coexisting } \\
\text { conditions }\end{array}$} \\
\hline $\begin{array}{l}\text { Diabetes } \\
\quad \text { mellitus (\%) }\end{array}$ & $32.7 \%, 33.0 \%$ & $19 \%, 17 \%$ & $31 \%, 26 \%$ & $18.7 \%, 23.2 \%$ & $36 \%, 31 \%$ & $26.1 \%, 25.3 \%$ \\
\hline$P$ value $\dagger$ & .95 & .80 & .72 & .44 & .48 & .976 \\
\hline $\begin{array}{l}\text { Hypertension } \\
(\%)\end{array}$ & $49.4 \%, 50.0 \%$ & $75 \%, 70 \%$ & $77 \%, 73 \%$ & $58.8 \%, 76.0 \%$ & $88 \%, 81 \%$ & $69.4 \%, 72.7 \%$ \\
\hline$P$ value $\dagger$ & .9 & .78 & .67 & .006 & .37 & .605 \\
\hline $\begin{array}{l}\text { Hyperlipidemia } \\
(\%)\end{array}$ & $29.3 \%, 30.1 \%$ & $65 \%, 60 \%$ & $53 \%, 47 \%$ & $70.0 \%, 69.0 \%$ & $74 \%, 72 \%$ & $62.4 \%, 72.1 \%$ \\
\hline$P$ value $\dagger$ & .84 & .78 & .43 & .89 & .85 & .09 \\
\hline \multicolumn{7}{|l|}{$\begin{array}{l}\text { Procedural } \\
\text { characteristics }\end{array}$} \\
\hline DES $(\%)$ & $71.1 \%$ & $35 \%$ & $100 \%$ & $100 \%$ & $100 \%$ & $59.9 \%$ \\
\hline $\begin{array}{l}\text { Distal LMCA } \\
\text { disease }(\%)\end{array}$ & $51.7 \%, 52.2 \%$ & $56 \%, 60 \%$ & $89 \%, 70 \%$ & $81.3 \%, \mathrm{NR}$ & $60 \%, \mathrm{NR}$ & $80.3 \%, 82.5 \%$ \\
\hline$P$ value $\dagger$ & .9 & .63 & $<.001$ & NR & NR & .742 \\
\hline
\end{tabular}


TABLE 1. Continued

\begin{tabular}{|c|c|c|c|c|c|c|}
\hline & $\begin{array}{c}\text { Seung } \\
\text { et } \mathrm{al}, \mathbf{2 0 0 8}^{3}\end{array}$ & $\begin{array}{c}\text { Buszman } \\
\text { et al, } \text { 2008 }^{2}\end{array}$ & $\begin{array}{c}\text { Palmerini } \\
\text { et al, } 2007^{4}\end{array}$ & $\begin{array}{c}\text { Chieffo } \\
\text { et al, 2006 }\end{array}$ & $\begin{array}{c}\text { Lee } \\
\text { et al, 2006 }\end{array}$ & $\begin{array}{c}\text { Palmerini } \\
\text { et al, 2006 }\end{array}$ \\
\hline $\begin{array}{l}\text { Off-pump } \\
\quad \text { CABG }(\%)\end{array}$ & $42 \%$ & $2 \%$ & $21 \%$ & $39.5 \%$ & $0 \%$ & $0 \%$ \\
\hline $\begin{array}{l}\text { Arterial } \\
\quad \text { conduit }(\%)\end{array}$ & $98.4 \%$ & $72 \% \#$ & $93 \% * *$ & NR & $96 \% \dagger \dagger$ & $94.2 \% * *$ \\
\hline \multicolumn{7}{|l|}{$\begin{array}{r}\text { Pharmacologic } \\
\text { treatment }\end{array}$} \\
\hline $\begin{array}{l}\text { Percutaneous } \\
\text { coronary } \\
\text { intervention } \\
\text { with stents }\end{array}$ & $\begin{array}{l}\text { Aspirin indefinitely }+ \\
\text { clopidogrel or } \\
\text { ticlopidine } \geq 1 \mathrm{mo} \\
\text { (BMS) or } \\
\text { clopidogrel } \geq 6 \mathrm{mo} \\
\text { (DES) }\end{array}$ & $\begin{array}{l}\text { Double } \\
\text { antiplatelet } \\
\text { treatment } \\
\quad \geq 1 \mathrm{y}\end{array}$ & $\begin{array}{l}\text { Aspirin indefinitely } \\
\text { + ticlopidine or } \\
\text { clopidogrel } \\
\text { 3-12 mo }\end{array}$ & NR & $\begin{array}{l}\text { Aspirin indefinitely }+ \\
\quad \text { clopidogrel } \geq 6 \mathrm{mo}\end{array}$ & NR \\
\hline CABG & NR & & & & NR & \\
\hline
\end{tabular}

When two values are given, the first represents percutaneous coronary intervention with stenting and the second represents coronary artery bypass grafting. IQR, Interquartile range; $N R$, not reported; $C C S$, Canadian Cardiovascular Society; $D E S$, drug-eluting stents; $L M C A$, left main coronary artery; $C A B G$, coronary artery bypass grafting; $B M S$, bare metal stent. *Median. $\dagger$ Reported in each individual study. $\nmid$ Propensity-matched patients from 1102 in the percutaneous coronary intervention with stenting group and 1138 in the coronary artery bypass grafting group. $\S \mathrm{P}=.97$. $\|$ Non-ST elevation. $\uparrow$ Non-Q wave. \#Left internal thoracic artery. **Internal thoracic artery. $\dagger \dagger$ Internal thoracic artery to left anterior descending coronary artery.

CABG group; and the durations of follow-up of the included studies were relatively short. In an attempt to correct for and minimize selection bias in nonrandomized observational studies, we pooled adjusted risk estimates. Nevertheless, these findings should be viewed in light of the need to wait for the results of ongoing large randomized, controlled trials (eg, COMBAT, SYNTAX, and REVASCULARIZE studies) before drawing any definitive conclusion.

\section{References}

1. Chieffo A, Morici N, Maisano F, Bonizzoni E, Cosgrave J, Montorfano M, et al. Percutaneous treatment with drug-eluting stent implantation versus bypass surgery for unprotected left main stenosis: a single-center experience. Circulation. 2006; $113: 2542-7$
2. Buszman PE, Kiesz SR, Bochenek A, Peszek-Przybyla E, Szkrobka I, Debinski M, et al. Acute and late outcomes of unprotected left main stenting in comparison with surgical revascularization. J Am Coll Cardiol. 2008;51:538-45.

3. Seung KB, Park DW, Kim YH, Lee SW, Lee CW, Hong MK, et al. Stents versus coronary-artery bypass grafting for left main coronary artery disease. $N$ Engl J Med. 2008;358:1781-92.

4. Palmerini T, Barlocco F, Santarelli A, Bacchi-Reggiani L, Savini C, Baldini E, et al. A comparison between coronary artery bypass grafting surgery and drug eluting stent for the treatment of unprotected left main coronary artery disease in elderly patients (aged $\geq 75$ years). Eur Heart J. 2007;28:2714-9.

5. Lee MS, Kapoor N, Jamal F, Czer L, Aragon J, Forrester J, et al. Comparison of coronary artery bypass surgery with percutaneous coronary intervention with drug-eluting stents for unprotected left main coronary artery disease. J Am Coll Cardiol. 2006; 47:864-70.

6. Palmerini T, Marzocchi A, Marrozzini C, Ortolani P, Saia F, Savini C, et al. Comparison between coronary angioplasty and coronary artery bypass surgery for the treatment of unprotected left main coronary artery stenosis (the Bologna Registry). Am J Cardiol. 2006;98:54-9. 
Review:

$\mathrm{PCl}-\mathrm{S}$ vs $\mathrm{CABG}$ for unprotected LMCA.

Comparison:

$01 \mathrm{PCl}-\mathrm{S}$ VS CABO

Outcome:

01 Death

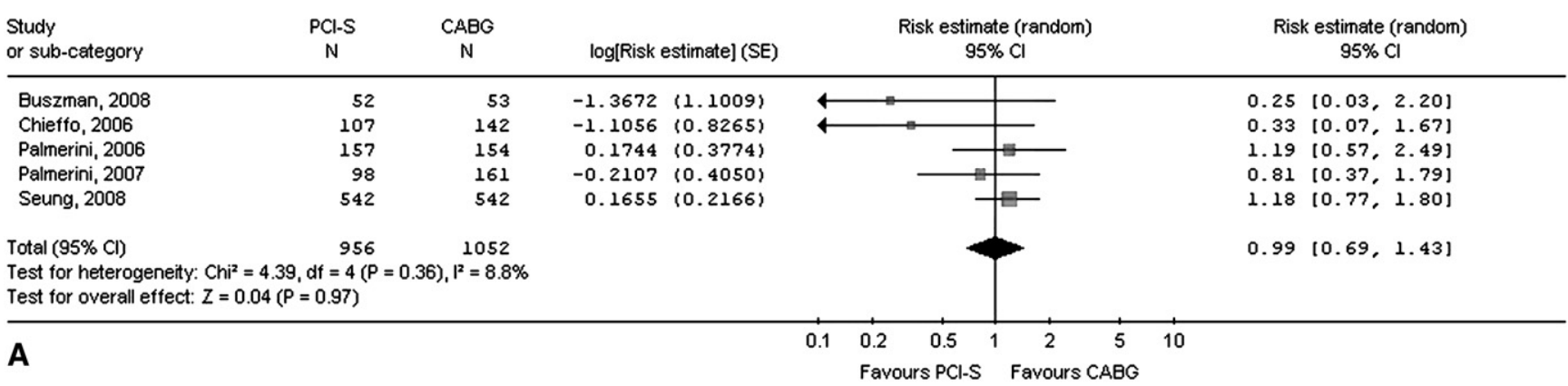

Review:

Comparison:

Outcome:

PCI-S vS CABG for unprotected LMCA

01 PCI-S vs CABG

02 Repeat revascularization

$\begin{array}{lc}\text { Study } & \mathrm{PCl}-\mathrm{S} \\ \text { or sub-category } & \mathrm{N}\end{array}$

Buszman, 2008

Chieffo, 2006

Palmerini, 2006

Seung, 2008

Total $(95 \% \mathrm{Cl})$

Test for heterogeneity: $\mathrm{Chi}^{2}=4.07, \mathrm{df}=3(\mathrm{P}$

Test for overall effect: $Z=6.37(P<0.00001)$

\section{B}

$\begin{array}{rrrr}52 & 53 & 1.1177 & (0.4781) \\ 107 & 142 & 1.4398 & (0.5820) \\ 157 & 154 & 2.5257 & (0.5388) \\ 542 & 542 & 1.5602 & (0.2713)\end{array}$

91 CABG

$\log [$ Risk estimate] (SE)

Risk estimate (random)

$95 \% \mathrm{Cl}$

Risk estimate (random)

$95 \% \mathrm{Cl}$

SE)

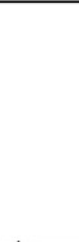

$\square$
$\square$
$\square$

$\begin{array}{ccccc}0.01 & 0.1 & 1 & 10 & 100 \\ & \text { Favours PCI-S } & \text { Favours CABG } & \end{array}$

$3.06[1.20,7.80]$

$4.22[1.35,13.20]$

$12.50[4.35,35.94]$

$4.76[2.80,8.10]$

$5.05[3.07,8.30]$

\begin{tabular}{|c|c|c|c|}
\hline $\begin{array}{l}\text { Review: } \\
\text { Comparison: } \\
\text { Outcome: }\end{array}$ & $\begin{array}{l}\text { PCI-S vS CABG for unprote } \\
01 \mathrm{PCI}-\mathrm{S} \text { VS } \mathrm{CABG} \\
03 \mathrm{MACCE}\end{array}$ & MCA & \\
\hline $\begin{array}{l}\text { Study } \\
\text { or sub-category }\end{array}$ & $\begin{array}{c}\mathrm{PCl}-\mathrm{S} \\
\mathrm{N}\end{array}$ & $\begin{array}{c}\text { CABG } \\
N\end{array}$ & log[Risk estimate] (SE) \\
\hline Buszman, 2008 & 52 & 53 & $0.2267(0.3183)$ \\
\hline Chieffo, 2006 & 107 & 142 & $-0.5656(0.4514)$ \\
\hline Lee, 2006 & 50 & 123 & $-1.0296(0.4958)$ \\
\hline $\begin{array}{l}\text { Total }(95 \% \mathrm{Cl}) \\
\text { Test for heteroge } \\
\text { Test for overall ef }\end{array}$ & $\begin{array}{l}209 \\
\text { eneity: } \mathrm{Chi}^{2}=5.20, \mathrm{df}=2(\mathrm{P} \\
\text { ffect: } \mathrm{Z}=0.99(\mathrm{P}=0.32)\end{array}$ & $\begin{array}{c}318 \\
1^{2}=61.6 \%\end{array}$ & \\
\hline
\end{tabular}

Test for overall effect: $Z=0.99(P=0.32)$

\section{C}

0.10

FIGURE 1. Outcomes and meta-analyses at follow-up for death (A), repeated revascularization (B), and major adverse cardiac and cerebrovascular events (MACCE; C). PCI-S, Percutaneous coronary intervention with stents; $C A B G$, coronary artery bypass grafting; $L M C A$, left main coronary artery; $C I$, confidence interval. 